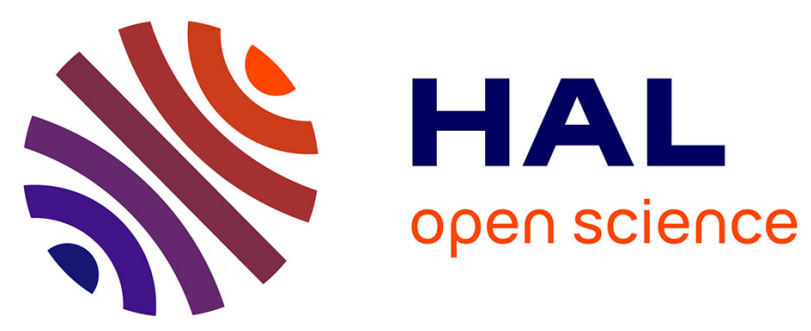

\title{
Reclassification of a TMC1 synonymous substitution as a variant disrupting splicing regulatory elements associated with recessive hearing loss
}

Christel Vaché, David Baux, Julie Bianchi, Corinne Baudoin, Valérie Faugère, Christine Francannet, Michel Koenig, Vasiliki Kalatzis, Anne-Françoise Roux

\section{To cite this version:}

Christel Vaché, David Baux, Julie Bianchi, Corinne Baudoin, Valérie Faugère, et al.. Reclassification of a TMC1 synonymous substitution as a variant disrupting splicing regulatory elements associated with recessive hearing loss. European Journal of Human Genetics, In press, 10.1038/s41431-021-01010-9 . hal-03470784

\section{HAL Id: hal-03470784 \\ https://hal.science/hal-03470784}

Submitted on 10 Dec 2021

HAL is a multi-disciplinary open access archive for the deposit and dissemination of scientific research documents, whether they are published or not. The documents may come from teaching and research institutions in France or abroad, or from public or private research centers.
L'archive ouverte pluridisciplinaire HAL, est destinée au dépôt et à la diffusion de documents scientifiques de niveau recherche, publiés ou non, émanant des établissements d'enseignement et de recherche français ou étrangers, des laboratoires publics ou privés. 


\title{
Reclassification of a TMC1 synonymous substitution as a variant disrupting splicing regulatory elements associated with recessive hearing loss
}

\author{
Christel Vaché ${ }^{1,2} \stackrel{\text { }}{ }$, David Baux ${ }^{1,2}$, Julie Bianchi ${ }^{1}$, Corinne Baudoin ${ }^{1}$, Valérie Faugère ${ }^{1}$, Christine Francannet $^{3}$, Michel Koenig $^{1}$, \\ Vasiliki Kalatzis ${ }^{2}$ and Anne-Françoise Roux ${ }^{1,2}$
}

\begin{abstract}
Alterations of the transmembrane channel-like 1 gene (TMC1) are involved in autosomal recessive and dominant nonsyndromic hearing loss (NSHL). To date, up to 117 causal variants including substitutions, insertions and splice variants have been reported in families from different populations. In a patient suffering from severe prelingual NSHL, we identified, in the homozygous state, the previously considered likely benign synonymous c.627C>T; p.(Leu209=) substitution. We used in silico tools predicting variantinduced alterations of splicing regulatory elements (SREs) and pinpointed this transition as a candidate splice-altering variation. Functional splicing analysis, using a minigene assay, confirmed that the variant altered a critical regulatory sequence which is essential for the exon 11 inclusion in the TMC1 transcripts. This result was reinforced by the analysis of orthologous TMC1 mammalian sequences for which the deleterious effect on the mRNA processing of a native thymidine was always counteracted by the presence of a stronger donor splice site or additional enhancer motifs. This study demonstrates, for the first time, the pathogenicity of the $c .627 \mathrm{C}>\mathrm{T}$ alteration leading to its reclassification as a causal variant impacting SREs and highlights the major importance of exhaustive studies to accurately evaluate the pathogenicity of a variant, regardless of the variation type.
\end{abstract}

\section{INTRODUCTION}

Hereditary nonsyndromic hearing loss (NSHL) is a disorder characterized by a large genetic heterogeneity with at least 123 causal genes (Hereditary Hearing Loss Homepage; https:// hereditaryhearingloss.org/; accessed on August 2021). Autosomal recessive NSHL (ARNSHL) is the most common form diagnosed in infancy and early childhood, accounting for $\sim 80 \%$ of cases, followed by autosomal dominant NSHL (ADNSHL) representing the remaining $20 \%$ in addition to rare $\mathrm{X}$-linked forms [1].

The transmembrane channel-like 1 gene (TMC1), encoding a major subunit of the sensory hair cells mechanoelectrical transduction channel, is implicated in both the AR- and ADNSHL forms DFNB7/11 and DFNA36, respectively [2]. DFNB7/11, which is mainly associated with congenital/prelingual severe to profound deafness, has been described in the worldwide population with a prevalence up to $8 \%$ in Turkish families presenting with ARSNHL without the prevalent GJB2 pathogenic variants [3]. To date up to 117 TMC1 pathogenic alterations, including only five variants associated with a dominant inheritance pattern of the disease, are reported in the Human Gene Mutation Database (HGMD Professional 2020.3; https://portal.biobase-international.com) and Leiden Open Variation Database (LOVD) Global Variome Shared Instance (LOVD GVShared, https://databases.lovd.nl/shared/genes/ TMC1). The causal variations are located throughout the gene and comprise substitutions, insertions, deletions and splice variants.
Among the 24 splice alterations listed in the HGMD database, 23 are located in introns. Eighteen of them abolish the AG/GT canonical splice sites, one disturbs a polypyrimidine tract [4], one generates an AG motif in an AG exclusion zone [2], another creates a new GT splice site [5] and three are located at the +5 or +3 key positions of the donor splice-site consensus sequence [5-7]. The only TMC1 exonic variant reported to have an effect on splicing is the $c .453 \mathrm{G}>\mathrm{A}$ transition, located at the last nucleotide of exon 9, which directly affects the natural donor splice-site sequence [8].

Exonic variants away from the acceptor or donor canonical splice sequences (NYAG/G and CAG/GUAAGU, respectively) can alter the splicing process through different mechanisms, such as creation or disruption of splicing regulatory elements (SREs). Thanks to the continual improvement of bioinformatics prediction programs and implementation of functional analyses in genetic laboratories, these deleterious alterations, including silent variants that impact exonic splice silencer (ESS) or enhancer (ESE) motifs, have increasingly been identified in various diseases [9-11].

In this article, using massively parallel sequencing (MPS) of a patient presenting with severe ARNSHL and a minigene splicing assay, we reclassified the previously reported, likely benign, silent TMC1 substitution c.627C>T; p.(Leu209=) as a pathogenic SREsdisrupting variation, and consequently tested the published neighboring alterations c.630_632delCAT [4] and c.624C>A [12]

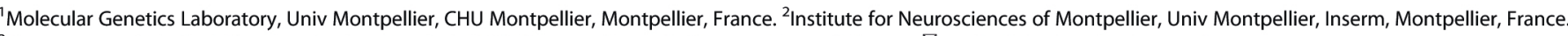

${ }^{3}$ Department of Medical Genetics, Estaing Hospital, CHU Clermont-Ferrand, Clermont-Ferrand, France. ${ }^{\infty}$ email: christel.vache@inserm.fr 
This study illustrates the critical importance of an exhaustive analysis of synonymous variants and more generally emphasizes the special attention that is needed to accurately classify variants that can alter splice regulatory motifs.

\section{SUBJECTS AND METHODS \\ Case presentation}

The proband investigated in this study was a child affected by apparent NSHL. A clinical questionnaire was completed by his parents concerning his family history and an audiologic assessment was performed to evaluate his degree of hearing loss in accordance with the $02 / 1$ bis recommendation of the International Bureau for Audiophonology (BIAP; https://www.biap. org/). Informed consent for genetic analyses was obtained from his father after explanation of the nature of the test and the possible implications for his son and his family. This study was performed in accordance with the French law on bioethics (revised July 7, 2011, number 2011-814). The experimental protocol was approved by the Montpellier University Hospital (CHU Montpellier) as part of the molecular diagnostic activity. The authorization number given by the Regional Health Agency (ARS) is LR/2013-No 190.

\section{DNA analysis}

Genomic DNA from the patient and his father was isolated from peripheral blood samples using standard procedures. The patient's DNA was analyzed by MPS using a hearing loss gene panel on an Illumina MiniSeq sequencer (Illumina, San Diego, CA, USA). The screened genes and the complete workflow used to identify pathogenic alterations have been already described [13]. This molecular diagnosis strategy included a copy number estimation of each region using the MobiCNV algorithm (https://github. $\mathrm{com} / \mathrm{mobidic}$ (MobiCNV) completed by a direct visualization of the sequenced reads with the Integrative Genomics Viewer (IGV) software (v2.7.2) [14].

Validation and familial segregation of the TMC1 variants were conducted by PCR-Sanger sequencing, with the forward primer 5'-GGACCAATGCCTCACAATTAA-3' combined with the reverse primer $5^{\prime}$-TCCTATGACTCTAAGACGTGA-3' and the forward primer 5'-GGAAGCTCAAACTGTGACAG-3' paired with the reverse primer $5^{\prime}$-CACTTACAAATTAGGTTGATATC- $3^{\prime}$, using the BigDye Terminator v3.1 cycle sequencing kit (Applied Biosystems, Courtaboeuf, France) on an Applied Biosystems $3500 \mathrm{Dx}$ Genetic Analyzer (Applied Biosystems).

\section{In vitro splicing analysis}

Minigene constructs were carried out as previously described [15]. Briefly, PCR-amplified DNA fragments containing the TMC1 exon 11 (107 bp) encompassed by $257 \mathrm{bp}$ of the $5^{\prime}$, and $291 \mathrm{bp}$ of $3^{\prime}$, intronic flanking sequences were generated from the patient and a control individual. The mutated (c.627T) and the corresponding wild-type (c.627C) pSPL3 minigenes were then constructed and a mutagenesis experiment with the QuikChange II Site-directed mutagenesis kit (Agilent, Courtaboeuf, France) was conducted to generate the mutated (c.630_632del) and (c.624C>A) minigenes. The four constructs were then transfected into HeLa cells using the FuGENE ${ }^{\circ} 6$ Transfection Reagent (Promega, Charbonnières-les-bains, France), according to the manufacturer's instructions. Forty-eight hours after transfection total RNA was extracted with the NucleoSpin RNA II isolation kit and reverse transcription was performed using the SuperScript ${ }^{T M}$ \|I Reverse Transcriptase (Invitrogen, Cergy-Pontoise, France). PCR amplifications were carried out with the SD6 and SA2 pSPL3-specific primers [16]. PCR products were sizeseparated on agarose gel and Sanger sequenced. Experiments were carried out in triplicate.

\section{In silico variant analysis}

Several DNA variation databases including HGMD, Genome Aggregation Database (gnomAD) (https://gnomad.broadinstitute.org/), Single Nucleotide Polymorphism Database (dbSNP) (https://www.ncbi.nlm.nih.gov/snp/), Clinical Variation Database (ClinVar) (https://www.ncbi.nlm.nih.gov/clinvar/), Deafness Variation Database (DVD) (https://deafnessvariationdatabase.org/), LOVD GVShared, the online variant interpretation platform MobiDetails [17] (https://mobidetails-iurc-montp-inserm.fr/MD/) have been interrogated to collect information on the variants. All last accessed in September 2021.

The effect of variants on SREs was predicted using two independent in silico approaches [18] based on the $\triangle \mathrm{tESR}_{\text {seq }}$ score change calculated via the HExoSplice web tool (bioinfo.univ-rouen.fr/HExoSplice_submit/index. php) and the $\Delta H Z_{E I}$ value provided by the HEXplorer algorithm (www2. hhu.de/rna/html/hexplorer_score.php). Previously published exon skipping and exon inclusion thresholds [19] of $-0.5,+0.5$ and $-20,+20$ were applied to the $\Delta \mathrm{tESRseq}$ scores and the $\Delta \mathrm{HZ}_{\mathrm{El}}$ values, respectively.

Nucleotide conservation among vertebrates of the human c.627C TMC1 residue was studied using the Multiz Alignment of 100 Vertebrates track of the University of California Santa Cruz Genome Browser-hg19 assembly tool [20] (https://genome.ucsc.edu/). Sequences of interest were then compared using the MaxEntScan score5ss tool [21] (http://hollywood.mit. edu/burgelab/maxent/Xmaxentscan_scoreseq.html) and the Exonic Splice Enhancers (ESE) finder 3.0 web-based resource [22] (http://krainer01.cshl. edu/cgi-bin/tools/ESE3/esefinder.cgi?process=home) by applying default settings.

\section{Variant description}

Nomenclature of the variants follows the Human Genome Variation Society [23] recommendations v20.05 (http://varnomen.hgvs.org/) with nucleotide +1 corresponding to the A of the ATG initiation codon in the TMC1 reference sequence NM_138691.2; NG_008213.1. The TMC1 c.627C>T variant (g.75366857C > T (GRCh37)) has been added to LOVD GVShared and reclassified using the web version of the InterVar tool [24] linked with MobiDetails, in accordance with the 2015 American College of Medical Genetics and Genomics-Association for Molecular Pathology (ACMG-AMP) guidelines [25]. The adapted ACMG/AMP guidelines for variant interpretation in the context of hearing loss [26] have also been used. The TMC1 c.$258 \mathrm{~A}>\mathrm{C}(\mathrm{g} .75231331 \mathrm{~A}>\mathrm{C}), \mathrm{c.624C}>\mathrm{A}(\mathrm{g} .75366854 \mathrm{C}>\mathrm{A})$ and c.630_632del (g.75366860_75366862del) variants have also been studied.

\section{RESULTS}

The family (S1993) presented in this study (Fig. 1A) was a consanguineous Turkish family with two generations including one affected 5-year-old boy (II-1). This proband did not receive an early neonatal hearing screen, but historical data reported that his parents worried about his audition from as early as 9 months old. At the age of 3 years, he had a significant language delay and a specialized consultation was carried out to test his audition. The audiometric assessment revealed a bilateral symmetric severe group one hearing loss on pure tone audiometry according the BIAP recommendations. When he was 4 years old, he was fitted with hearing aids and his language evolved favorably. Air conduction without hearing aids is reported for patient II-1 (5 years old) in Fig. 1B.

MPS performed on the DNA of the proband did not identify clear pathogenic variations in the genes of the panel but detected two very rare variants in the TMC1 gene, namely the $5^{\prime}$ UTR c.$258 \mathrm{~A}>\mathrm{C}$ transversion and the synonymous c.627C $>\mathrm{T}$, p. (Leu209=) exon 11 substitution, both in the homozygous state. Presence of a large deletion in trans of the variants was excluded by an exoncopy-number evaluation and a direct visualization of the reads using the IGV tool. Sanger sequencing (Fig. 1C) confirmed the homozygosity of the two variations in patient II-1 and established the heterozygous status of his father (I-1).

The c.-258A $>C$ variation, located in the noncoding exon 3 of TMC1, was already archived in dbSNP (rs937270834) with a reported frequency varying between $0.0008 \%$ (TOPMED) and $0.007 \%$ (ALFA). Based on predicted alterations of the TMC1 RNA folding, this alteration was initially considered as the most probable causative variant in two ethnically related Iranian families suffering from ARNSHL [27]. It was described as likely pathogenic in DVD, pathogenic or of uncertain significance in LOVD GVShared. It was also reported in HGMD but not in ClinVar.

The c.627C>T, p.(Leu209=) variation was present in gnomAD, always in the heterozygous state, with a $0.003 \%$ allele frequency for the total population. It was identified in dbSNP (rs748580616), reported as likely benign in ClinVar and classified as a variant of uncertain significance (VUS) in DVD. It was absent from the HGMD and LOVD GVShared databases. Alignment of vertebrate DNA sequences surrounding the human c.627 TMC1 nucleotide revealed that this position was not constrained with a genomic 
TMC1

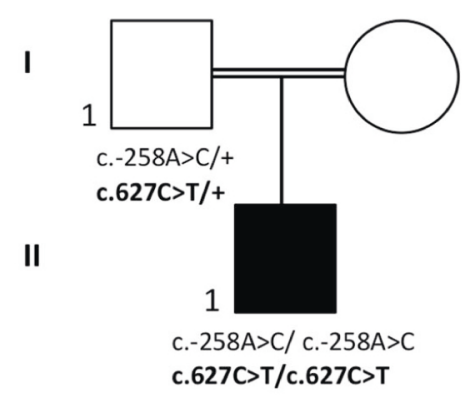

Frequency in Hertz $(\mathrm{Hz})$

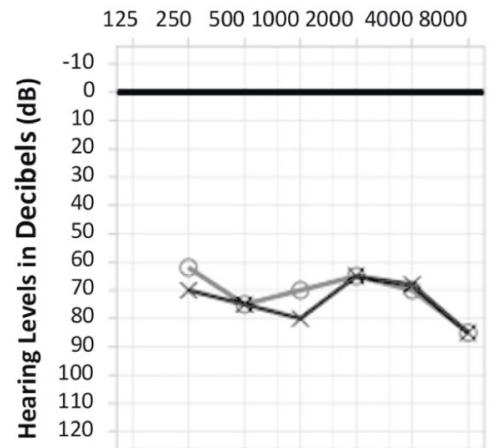

C

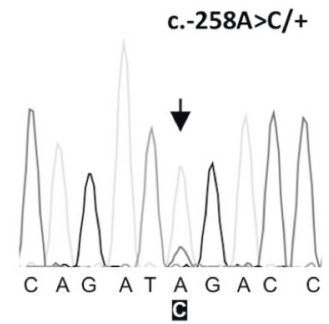

c. $-258 \mathrm{~A}>\mathrm{C} / \mathrm{c} .-258 \mathrm{~A}>\mathrm{C}$

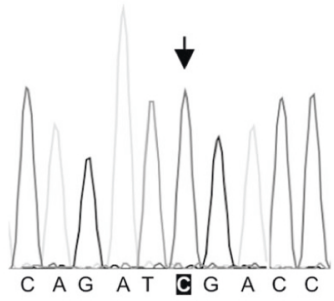

|-1

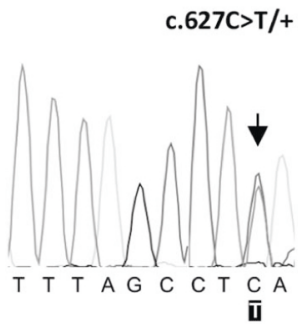

c. $627 \mathrm{C}>\mathrm{T} / \mathrm{c} .627 \mathrm{C}>\mathrm{T}$

II-1

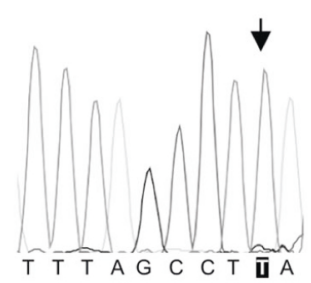

Fig. 1 Pedigree chart of the S1993 family, segregation of the TMC1 variants and audiogram of the proband. A Pedigree of the family with segregation of the TMC1 variants. B Audiogram of the patient at the age of 5 years old showing bilateral severe hearing loss. Scores for the right and left ears are symbolized by circles and crosses, respectively. C Sanger sequencing electropherograms of the C.-258A $>C$ and c.627C $>T$ TMC1 alterations for the heterozygous nonaffected father (I-1) and the homozygous patient (II-1). The c.-258A nucleotide is preferentially amplified in I-1. Variants are indicated with white bold font and highlighted in black.

evolutionary rate profiling score of 1.48 and that a thymidine was naturally present in 24 organisms. Located 16-bp upstream of the exon 11 natural donor splice site, the c.627 variation did not affect the consensus $5^{\prime}$ splice-site sequence and bioinformatic tools predicted that it did not create/activate new acceptor or donor splice sites. Based on these data, the silent variant was previously classified, by automated scoring using the web version of the InterVar bioinformatics software, as likely benign with the following 2015 ACMG-AMP criteria: PM2, BP4 and BP7. In accordance with this classification, the $c .627 \mathrm{C}>\mathrm{T}$ substitution, also detected in cis of the c.-258A $>C$ variation in the large Iranian families cited above, was not considered as a pathogenic candidate variant by the authors [27].

However, with our additional in silico analysis, the two independent SRE-dedicated approaches indicated a possible default of the splicing process by the variant via the disruption of SREs ( $\Delta \mathrm{tESR}$ seq score: $-1.31 ; \Delta \mathrm{HZ} \mathrm{El}_{\mathrm{El}}$ value: -60.13 ) leading to a potential increase of exon skipping (Fig. 2A; Supplementary Fig. 1). In order to test this hypothesis, a minigene assay based on the pSPL3 exon-trapping vector was conducted (Fig. 2B). Whereas the wild-type construct led to the synthesis of spliced transcripts with inclusion of $T M C 1$ exon 11, the $c .627 C>T$ mutant minigene induced the production of two populations of transcripts supporting the in silico predictions. Sanger sequencing of the RT-PCR products established the presence of a major population composed of transcripts lacking the $107 \mathrm{bp}$ of the TMC1 exon (r.536_642del) and a minor population consisting of correctly spliced mutated transcripts (accurate use of the $3^{\prime}$ and $5^{\prime}$ splice sites of the TMC1 exon $11 ; r .627 c>u$ ). The out-of-frame exon skipping was predicted to result in a prematurely truncated TMC1 protein (p.Ser179llefsTer12), if synthetized.

Because the presence of a thymidine in various organisms at the corresponding human c.657 position might seem incompatible with the mis-splicing event observed in our minigene assay, 


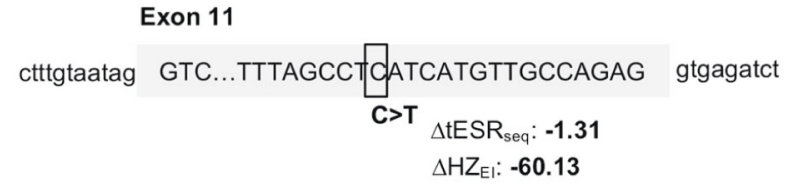

B

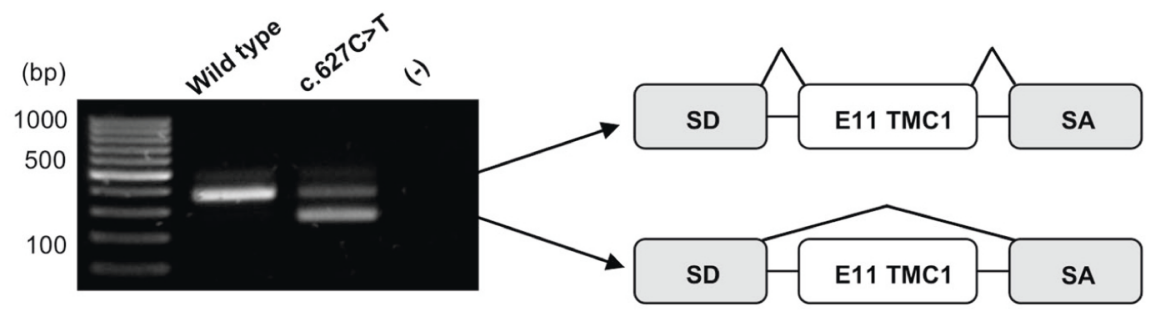

C
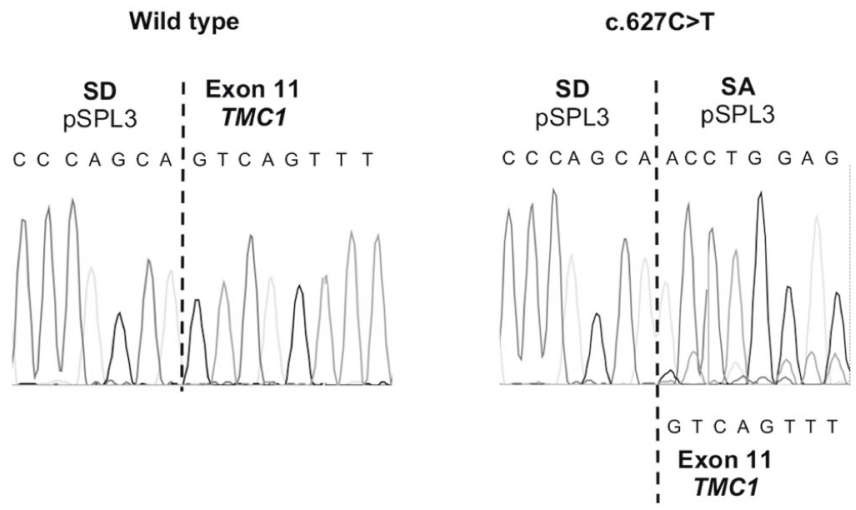

Fig. 2 Functional assay of the $\mathbf{c . 6 2 7 C}>\mathrm{T}$ TMC1 substitution. A Localization of the variant within exon 11 . The $\Delta \mathrm{tESR} \mathrm{R}_{\mathrm{seq}}$ and $\Delta \mathrm{HZ} \mathrm{El}_{\mathrm{El}} \mathrm{SCores}$ are indicated with bold font when they exceed the previously established thresholds. B Agarose gel electrophoresis of the RT-PCR products and schematic representation of the two populations of transcripts. C Sanger sequencing electropherograms of the RT-PCR products.

complementary in silico investigations were then conducted on some mammalian sequences harboring this nucleotide (Fig. 3). Interestingly, all studied organisms, except Opossum, had a stronger $5^{\prime}$ splice-site strength score than the one estimated for human (MaxEnt scores:10.06 and 7.66 respectively) due to a $G>A$ +3 nucleotide mismatch (Fig. 3A). Furthermore, Opossum who had the same $5^{\prime}$ splice-site strength score as predicted for human presented a very different distribution of ESE elements in comparison with the wild-type or mutated human sequence (Fig. 3B), with a significant concentration of serine and argininerich (SR) protein-binding motifs at the $3^{\prime}$ end of the exon.

Due to their respective positions, close to the c.627 variation, the c.630_632del and c.624C>A alterations, both previously identified in trans of pathogenic variants in patients suffering from ARNSHL, were tested using minigene assays to evaluate their impact on splicing. The c.630_632del alteration was not considered as a candidate SRE-disrupting variant by the HExoSplice tool ( $\triangle \mathrm{tESR}$ seq score: 0 ) but was predicted as a variant increasing exon 11 skipping by the HEXplorer algorithm $\left(\Delta \mathrm{HZ} Z_{\mathrm{El}}\right.$ value: -43.76) (Fig. 4A; Supplementary Fig. 2). Introduction of the deletion in our minigene system led to the production of two CDNA populations: a predominant one comprising accurately spliced mutated transcripts (r.630_632del) and a minor one with aberrant transcripts (r.536_642del) corresponding to the skipping of the TMC1 exon (Fig. 4B). Furthermore, it is noteworthy that additional ESE elements (SRSF1, SRSF1(IgM-BRCA1), SRSF2 and
SRSF5) were identified by the ESEfinder tool in the presence of the deletion (Fig. 4C). The c.624C>A transversion was not considered as a variant resulting in exon 11 skipping by the two SREpredictors $\left(\Delta \mathrm{tESR}_{\text {seq }}\right.$ score: $+0.51 ; \Delta \mathrm{HZ}_{\mathrm{El}}$ value: -9.82$)$ (Fig. $5 \mathrm{~A}$; Supplementary Fig. 3 ). As expected, the splicing minigene assay demonstrated that this substitution did not result in the exclusion of the TMC1 exon 11 in the synthetized mutated transcripts (r.624C>a) (Fig. 5B) and ESEfinder did not identify new SR proteinbinding sites (Fig. 5C).

\section{DISCUSSION}

The present study reports the exhaustive molecular diagnostic approach used to investigate the consequences of a synonymous TMC1 variation carried in the homozygous state by a young patient suffering from ARNSHL.

The c.627C>T; p.(Leu209=) TMC1 variant identified in the proband was recorded in ClinVar and was classified as likely benign following the 2015 ACMG-AMP recommendations in regard to its extremely low frequency in the total population (PM2), the presence of multiple lines of computational evidence suggesting no impact on gene or gene product (BP4), the absence of predicted splice defects associated with the splice consensus sequences or the creation of a new splice site, and a nucleotide position 627 that is not highly conserved (BP7). When the adapted ACMG/AMP guidelines for variant 


\begin{tabular}{|c|c|c|}
\hline Mut c.627 C>T & AGCCपTATCATGTTGCCAGAGgtgaga & 7.66 (5'ss \\
\hline Human & AGCСTCATCATGTTGCCAGAGgtgaga & 7.66 \\
\hline Alpaca & AGCСTтATCATGTTGCCAGAGgtaaga & 10.06 \\
\hline Cow & AGCCTTATCATGTTGCCAGAGgtaaga & 10.06 \\
\hline Sheep & AGCCTTATCATGTTGCCAGAGgtaaga & 10.06 \\
\hline Goat & AGCCTTATCATGTTGCCAGAGgtaaga & 10.06 \\
\hline Pig & AGCCTTATCATGTTACCAGAGgtaaga & 10.06 \\
\hline Opossum & AGCСTTATTATGTTACCGGAGgtgaga & 7.66 \\
\hline
\end{tabular}
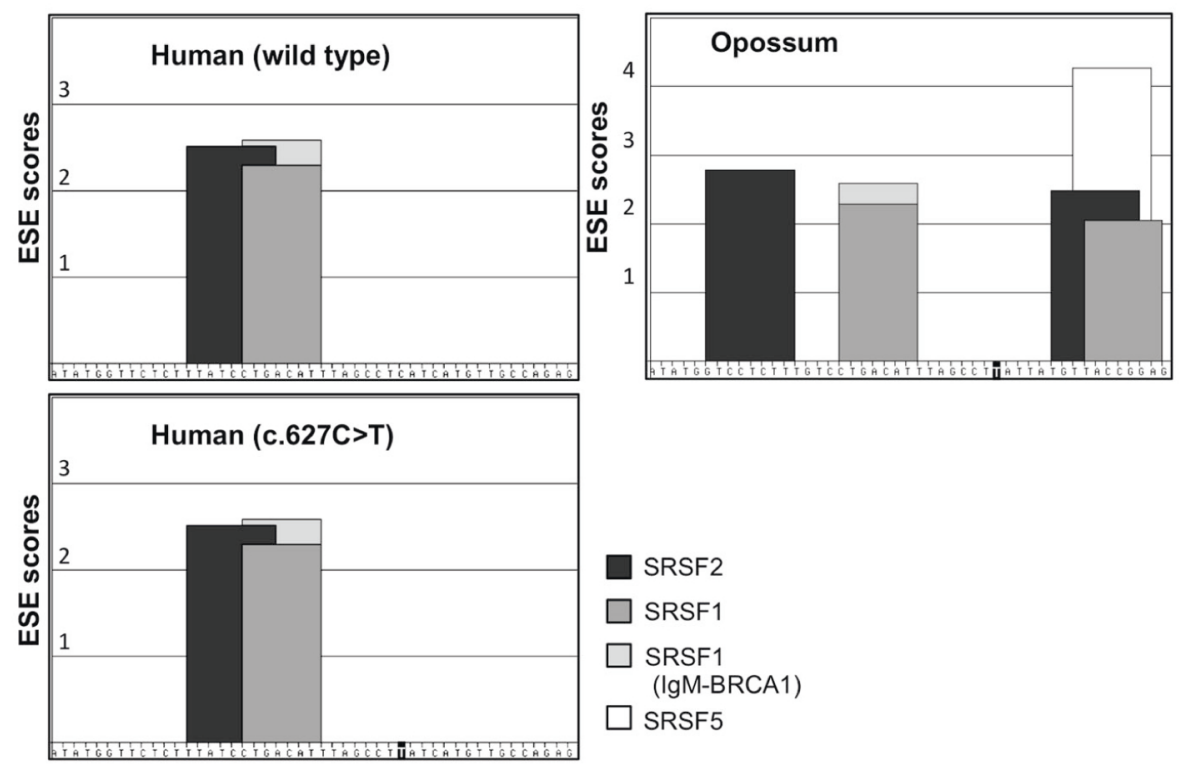

SRSF2

SRSF1

SRSF1

(IgM-BRCA1)

$\square$ SRSF5

Fig. 3 Analysis of the sequence context around the c.627C>T TMC1 variant among different mammals. A Alignments of mammalian TMC1 sequences carrying a native thymidine (bold black font) corresponding to the human $c .627 \mathrm{C}>\mathrm{T}$ variant. Differences with the human sequence are highlighted in gray. MaxEnt Donor splice-site strength scores (5'ss scores) are indicated. B Localizations and scores of putative binding motifs for the human serine/arginine-rich (SR) proteins (SRSF1, SRSF1(IgM-BRCA1), SRSF2 and SRSF5) predicted by the ESEfinder 3.0 tool in the human (wild type and c.627C >T mutated) and Opossum sequences. The shown DNA sequence on the $x$-axis corresponds to the last 47 bp of the studied exon. The c.627T nucleotide is indicated with white bold font and highlighted in black.

interpretation in the context of hearing loss were applied, this variant was classified as a VUS with two benign supporting criterions (BP4 and BP7) and two criterions in favor of pathogenicity (PM2 and PM3-supporting related to the homozygous occurrence of the variant due to consanguinity in the Iranian families). In this study, we predicted by in silico analyses that this transversion was a candidate splice-disrupting variant by alteration of SREs, thus recusing the previous BP4 and BP7 categorizations and supporting a deleterious effect (PP3). The previous likely benign classification of the variant with the BP7 criteria was mainly supported by a nucleotide position that was not highly conserved between species and the presence of the thymidine in different organisms. Our in silico analysis of splicing elements identified in exons carrying the thymidine offers a new interpretation of these data. Indeed, when native in mammals, the thymidine, which impacts the mRNA processing as demonstrated here, is systematically associated with other substitutions that could offset its negative effect by strongly increasing the strength of the natural donor splice site or by creating a new set of ESE motifs near the donor site. Consequently, in our point of view, the presence of the thymidine in other organisms should not be considered for its own but with its sequence environment that supports a pathogenic criterion for the classification of the human c.627C >T variation. For this reason, we manually adjusted its interVar interpretation by adding a new criterion of supporting pathogenicity (PP6) proposed to users in the WinterVar interface. Furthermore, because this very rare splice-altering TMC1 variant was identified in the homozygous state in other patients, from different families, presenting with ARNSHL linked to the DFNB7/ 11 locus, we decided similarly to add a new user-evidence criterion of moderate pathogenicity (PM7, in accordance with the supporting-PM3 criteria of the hearing loss ACMG/AMP guidelines) to precise the clinical interpretation of the variant. Finally, as a major result of our in vitro demonstration of the damaging effect of the substitution on the mRNA processing, we were able to assign a strong pathogenic criterion (PS3) to the variant.

In conclusion, taking into consideration all of these criteria we propose to reclassify the $c .627 \mathrm{C}>\mathrm{T}$ alteration as a class $\mathrm{V}$ 


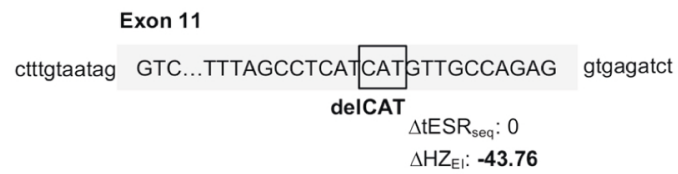

B

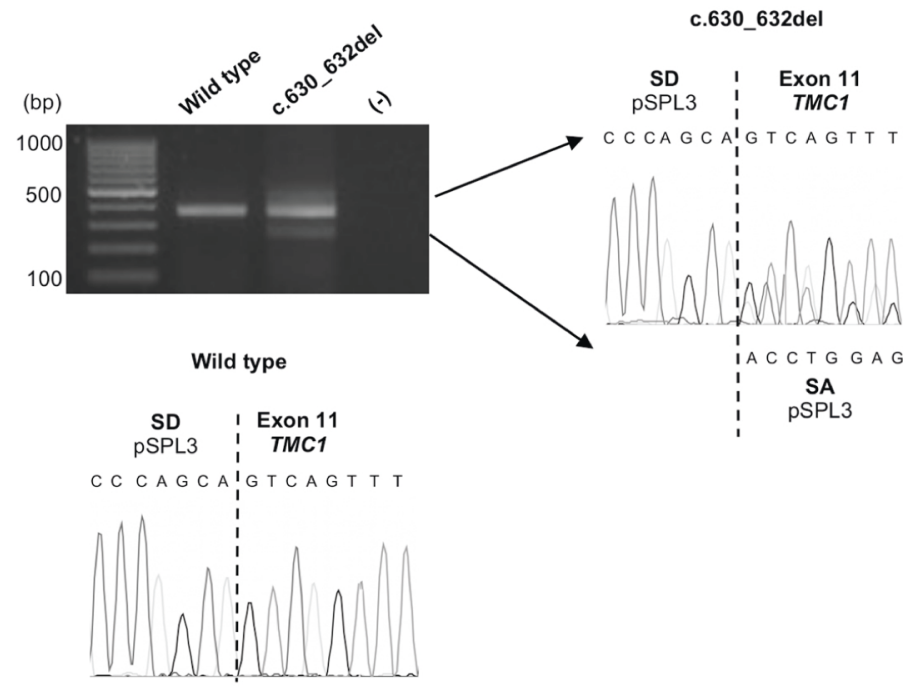

C

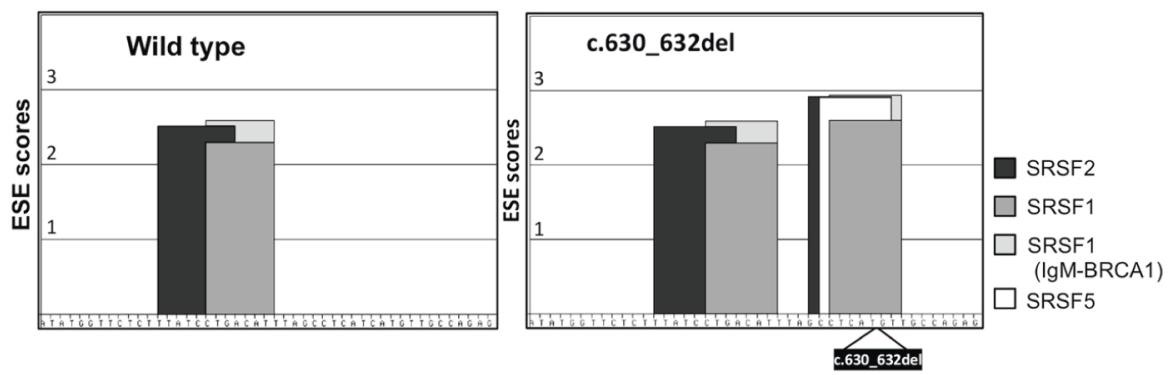

Fig. 4 Functional assay of the $\mathbf{c . 6 3 0} 632 \mathrm{del} T M C 1$ alteration. $A$ Localization of the variant within exon 11 . The $\Delta t E S R_{\text {seq }}$ and $\Delta H Z_{E I} S C O r e s$ are indicated with bold font when they exceed the previously established thresholds. B Agarose gel electrophoresis of the RT-PCR products and Sanger sequencing electropherograms of the RT-PCR products for the wild-type and mutated constructs. C Localizations and scores of putative binding motifs for the human serine/arginine-rich (SR) proteins (SRSF1, SRSF1(IgM-BRCA1), SRSF2 and SRSF5) predicted by the ESEfinder 3.0 tool in the wild-type and c.630_632del sequences. The shown DNA sequence on the $x$-axis corresponds to the last 47 bp of the TMC1 exon 11. The deletion is indicated above the sequence with white bold font and highlighted in black.

pathogenic variant and consider the c.-258A $>C$ substitution as VUS.

In addition, we also demonstrated the deleterious effect of the c.630_632del alteration on the splicing process with, in this case, a moderate impact. As already described by Tubeuf et al. [18], the HEXplorer score differences $\Delta \mathrm{HZ}_{\mathrm{EI}}$ variant-reference calculated for the $\mathrm{C.627C}>\mathrm{T}$ and the $\mathrm{C.630} 632$ del variations $(\Delta \mathrm{HZ} \mathrm{El}:-60.13$ and -43.76 , respectively) were correlated with the severity of the resulting splicing defect observed using minigene assays.

Results obtained from the different in silico tools used in this study point out the importance of taking into consideration a set of predictors, based on different matrices, to improve predictive value. For instance, the ESEfinder tool just provides scores for a limited number of dedicated ESE motifs (SRSF1 (heptamer), SRSF2 (octamer), SRSF5 (heptamer) and SRSF6 (hexamer)) among a multitude of potential SREs and was not able to predict the splicing defect caused by the $c .627 \mathrm{C}>\mathrm{T}$ variation, unlike the other two predictors used in this study. On the other hand, this tool was relevant to provide a different view of the sequence context and predicted new ESEs in presence of the c.630_632del variation that could be involved in an ESS/ESE balance in favor of a moderate exon skipping. In any case, bioinformatic predictions should be confirmed in functional studies, especially when SREs are potentially involved since they are not all functional, although predicted. The most effective method to determine the effect of a variant on the splicing process is to analyze mRNA from a relevant patient tissue or cell line established from patient's cells, but if not possible, a splicing reporter minigene assay can be used. This alternative approach is a rapid and accurate method included in our diagnostic strategy [13]. However, this test can present limitations mainly related to the construction of an artificial hybrid gene and the culture of transfected cells that may not exhibit the same pattern of cis-regulatory elements than in vivo. In our study the wild-type TMC1 exon 11 construct was always correctly processed, thus validating the approach and ensuring that the aberrant transcripts observed with the mutated constructs were due to the variants.

Numerous synonymous variants located away from the consensus splice-site sequences are often directly classified as 

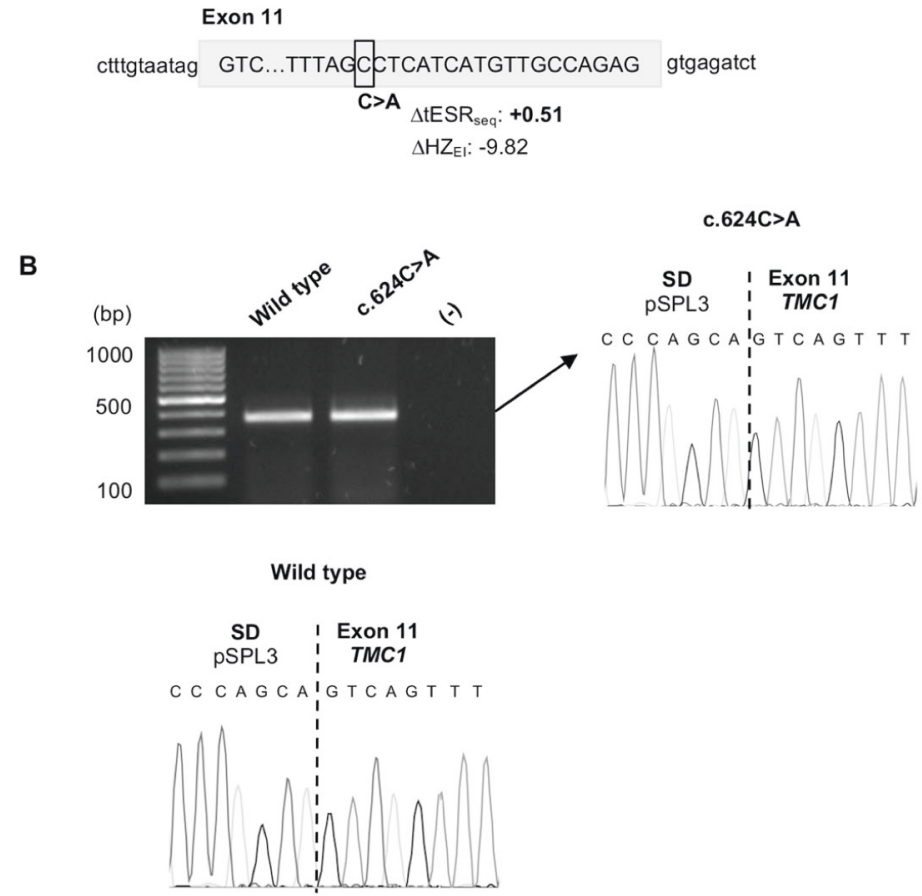

C

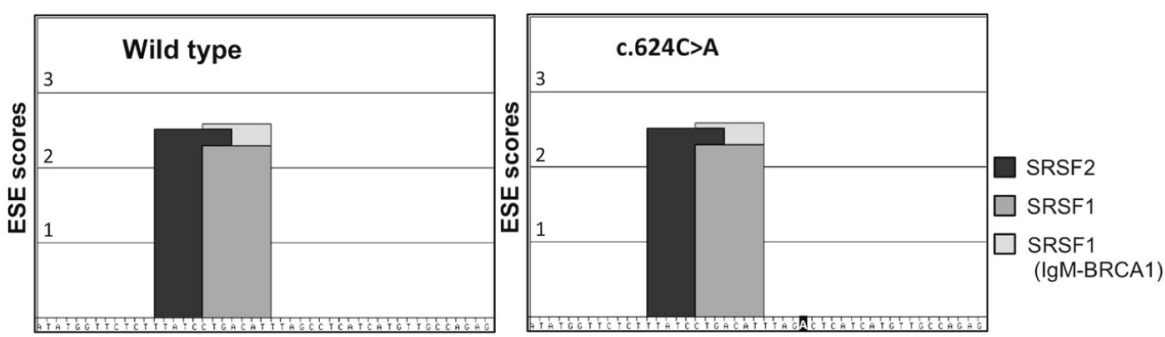

Fig. 5 Functional assay of the $\mathbf{c . 6 2 4 C}>\mathbf{A}$ TMC1 variant. A Localization of the substitution within exon 11 . The $\Delta \mathrm{tESR}$ seq and $\Delta H Z_{\mathrm{El}} \mathrm{SCOres}$ are indicated with bold font when they exceed the previously established thresholds. B Agarose gel electrophoresis of the RT-PCR products and Sanger sequencing electropherograms of the RT-PCR product for the wild-type and mutated constructs. C Localizations and scores of putative binding motifs for the human serine/arginine-rich (SR) proteins (SRSF1, SRSF1(IgM-BRCA1) and SRSF2) predicted by the ESEfinder 3.0 tool in the wild-type and c.624C>A sequences. The shown DNA sequence on the $x$-axis corresponds to the last 47 bp of the $T M C 1$ exon 11 . The $c .624 A$ nucleotide is indicated with white bold font and highlighted in black.

benign, especially if they are native in other organisms. To our knowledge, we report here the first investigation of a TMC1 silent variant leading to its reclassification as a pathogenic variation via the disruption of SREs. This result will inevitably have direct implications for the patients and their families in terms of medical management and genetic counseling but will be also of interest for diagnostic laboratories by emphasizing the critical importance of exhaustive studies regardless of the variation.

\section{DATA AVAILABILITY}

The data that support this study are available from the corresponding author upon reasonable request. The variants, individual and phenotype described in this manuscript are available on the LOVD GVShared, Individual ID number \#00383282.

\section{REFERENCES}

1. Morton NE. Genetic epidemiology of hearing impairment. Ann N Y Acad Sci. 1991;630:16-31.

2. Kurima K, Peters LM, Yang Y, Riazuddin S, Ahmed ZM, Naz S, et al. Dominant and recessive deafness caused by mutations of a novel gene, TMC1, required for cochlear hair-cell function. Nat Genet. 2002;30:277-84.
3. Sirmaci A, Duman D, Oztürkmen-Akay $H$, Erbek S, Incesulu A, Oztürk-Hişmi B, et al Mutations in TMC1 contribute significantly to nonsyndromic autosomal recessive sensorineural hearing loss: a report of five novel mutations. Int J Pediatr Otorhinolaryngol. 2009;73:699-705.

4. Ganapathy A, Pandey N, Srisailapathy CRS, Jalvi R, Malhotra V, Venkatappa M, et al. Non-syndromic hearing impairment in India: high allelic heterogeneity among mutations in TMPRSS3, TMC1, USHIC, CDH23 and TMIE. PLoS ONE. 2014;9: e84773.

5. Shafique S, Siddiqi S, Schraders M, Oostrik J, Ayub H, Bilal A, et al. Genetic spectrum of autosomal recessive non-syndromic hearing loss in Pakistani families. PLoS ONE. 2014;9:e100146.

6. Meyer CG, Gasmelseed NM, Mergani A, Magzoub MMA, Muntau B, Thye T, et al. Novel TMC1 structural and splice variants associated with congenital nonsyndromic deafness in a Sudanese pedigree. Hum Mutat. 2005;25:100.

7. de Heer A-MR, Collin RWJ, Huygen PLM, Schraders M, Oostrik J, Rouwette M, et al. Progressive sensorineural hearing loss and normal vestibular function in a Dutch DFNB7/11 family with a novel mutation in TMC1. Audio Neurootol. 2011;16:93-105

8. Sloan-Heggen CM, Babanejad M, Beheshtian M, Simpson AC, Booth KT, Ardalani $\mathrm{F}$, et al. Characterising the spectrum of autosomal recessive hereditary hearing loss in Iran. J Med Genet. 2015;52:823-9.

9. da Palma MM, Motta FL, Gomes CP, Salles MV, Pesquero JB, Sallum JMF. Synonymous variant in the $\mathrm{CHM}$ gene causes aberrant splicing in choroideremia. Investig Ophthalmol Vis Sci. 2020;61:38. 
10. Hu G, Zeng J, Wang C, Zhou W, Jia Z, Yang J, et al. A synonymous variant C.579A > $G$ in the ETFDH gene caused exon skipping in a patient with late-onset multiple Acyl-CoA dehydrogenase deficiency: a case report. Front Pediatr. 2020;8:118.

11. Sylvester B, Brindopke F, Suzuki A, Giron M, Auslander A, Maas RL, et al. A synonymous exonic splice silencer variant in IRF6 as a novel and cryptic cause of non-syndromic cleft lip and palate. Genes. 2020;11:E903.

12. He L, Pang $X$, Liu $H$, Chai $Y, W u ~ H$, Yang T. Targeted next-generation sequencing and parental genotyping in sporadic Chinese Han deaf patients. Clin Genet. 2018;93:899-904.

13. Baux D, Vaché $C$, Blanchet $C$, Willems M, Baudoin C, Moclyn M, et al. Combined genetic approaches yield a $48 \%$ diagnostic rate in a large cohort of French hearing-impaired patients. Sci Rep. 2017;7:16783.

14. Thorvaldsdóttir H, Robinson JT, Mesirov JP. Integrative Genomics Viewer (IGV): high-performance genomics data visualization and exploration. Brief Bioinform. 2013;14:178-92.

15. Le Guédard-Méreuze $S$, Vaché $C$, Baux D, Faugère $V$, Larrieu L, Abadie $C$, et al. Ex vivo splicing assays of mutations at noncanonical positions of splice sites in USHER genes. Hum Mutat. 2010;31:347-55.

16. Bottillo I, De Luca A, Schirinzi A, Guida V, Torrente I, Calvieri S, et al. Functional analysis of splicing mutations in exon 7 of NF1 gene. BMC Med Genet. 2007;8:4.

17. Baux D, Van Goethem C, Ardouin O, Guignard T, Bergougnoux A, Koenig M, et al. MobiDetails: online DNA variants interpretation. Eur J Hum Genet. 2021;29:356-60.

18. Tubeuf $H$, Charbonnier $C$, Soukarieh $O$, Blavier $A$, Lefebvre A, Dauchel $H$, et al. Large-scale comparative evaluation of user-friendly tools for predicting variantinduced alterations of splicing regulatory elements. Hum Mutat. 2020;41:1811-29.

19. Soukarieh $O$, Gaildrat $P$, Hamieh M, Drouet A, Baert-Desurmont S, Frébourg T, et al. Exonic splicing mutations are more prevalent than currently estimated and can be predicted by using in silico tools. PLoS Genet. 2016;12:e1005756.

20. Kent WJ, Sugnet CW, Furey TS, Roskin KM, Pringle TH, Zahler AM, et al. The human genome browser at UCSC. Genome Res. 2002;12:996-1006.

21. Yeo G, Burge CB. Maximum entropy modeling of short sequence motifs with applications to RNA splicing signals. J Comput Biol. 2004;11:377-94.

22. Cartegni L, Wang J, Zhu Z, Zhang MQ, Krainer AR. ESEfinder: a web resource to identify exonic splicing enhancers. Nucleic Acids Res. 2003;31:3568-71.

23. den Dunnen JT, Dalgleish R, Maglott DR, Hart RK, Greenblatt MS, McGowanJordan J, et al. HGVS Recommendations for the Description of Sequence Variants: 2016 Update. Hum Mutat. 2016;37:564-9.

24. Li Q, Wang K. InterVar: clinical interpretation of genetic variants by the 2015 ACMG-AMP guidelines. Am J Hum Genet. 2017;100:267-80.

25. Richards S, Aziz N, Bale S, Bick D, Das S, Gastier-Foster J, et al. Standards and guidelines for the interpretation of sequence variants: a joint consensus recommendation of the American College of Medical Genetics and Genomics and the Association for Molecular Pathology. Genet Med. 2015;17:405-24.

26. Oza AM, DiStefano MT, Hemphill SE, Cushman BJ, Grant AR, Siegert RK, et al. Expert specification of the ACMG/AMP variant interpretation guidelines for genetic hearing loss. Hum Mutat. 2018;39:1593-613.
27. Davoudi-Dehaghani E, Zeinali S, Mahdieh N, Shirkavand A, Bagherian H, Tabatabaiefar MA. A transversion mutation in non-coding exon 3 of the TMC1 gene in two ethnically related Iranian deaf families from different geographical regions; evidence for founder effect. Int J Pediatr Otorhinolaryngol. 2013;77:821-6.

\section{ACKNOWLEDGEMENTS}

The authors are grateful to the patient and his father who contributed to this study.

\section{AUTHOR CONTRIBUTIONS}

$\mathrm{CV}$ and $\mathrm{A}-\mathrm{FR}$ contributed to the conception and the design of the study. CV, JB, CB and VF performed the experiments. CV, A-FR, JB and CB analyzed and interpreted the data. $C F$ requested molecular diagnosis for the patient and provided patients' clinical data. CV wrote the first draft of the manuscript. A-FR, VK, DB and MK revised the manuscript. All authors contributed to the article and approved the submitted version.

\section{COMPETING INTERESTS}

The authors declare no competing interests.

\section{ETHICS APPROVAL AND CONSENT TO PARTICIPATE}

This study was performed in accordance with the French law on bioethics (revised July 7, 2011, number 2011-814). The experimental protocol was approved by the Montpellier University Hospital (CHU Montpellier) as part of the molecular diagnostic activity. The authorization number given by the Regional Health Agency (ARS) is LR/ 2013-No 190. 